\title{
Extracting SUSY parameters from LHC measurements using Fittino
}

\author{
K. Desch*, M. Uhlenbrock, P. Wienemann \\ University of Bonn, Bonn, Germany \\ E-mail: deschephysik.uni-bonn.de

\section{P. Bechtle}

Deutsches Elektronen-Synchrotron DESY, Hamburg, Germany

\begin{abstract}
With the start of the LHC, low-energy Supersymmetry can be probed over the largest part of the theoretically motivated parameter space. We investigate how well the parameters of the MSSM as well as of constrained models such as minimal Supergravity and Gauge Mediated SUSY breaking can be determined already from existing low-energy measurements such as $(g-2)_{\mu}$, rates of rare B- and K-meson decays and cosmological constraints as well as the dark matter density in the Universe. We then study the parameter precision as a function of the integrated luminosity ranging from $1 \mathrm{fb}^{-1}$ to $300 \mathrm{fb}^{-1}$ at the LHC. We also study the impact of a future linear collider to achieve precision measurements. The theoretical predictions are provided by the codes combined in the MasterCode and SPheno packages. Special emphasis is given to a careful evaluation of the stability of a global $\chi^{2}$ parameter estimation technique encoded in the Fittino package. This technique is based on the simulated annealing algorithm for global function minimization with error determination from repeated Monte Carlo experiments ("toy fits"). The method is augmented by an efficient scan of the $\chi^{2}$ hyper-surface in the multi-dimensional parameter space using a Markov-Chain-Monte-Carlo approach.
\end{abstract}

The 2009 Europhysics Conference on High Energy Physics,

July 16 - 222009

Krakow, Poland

${ }^{*}$ Speaker. 


\section{Introduction and Method}

In order to confront a model for physics beyond the Standard Model ("New Physics") with data from existing and future experiments, tools for a global comparison of the data with model predictions as a function of its parameters have to be employed. Fittino [1], 2] is such a tool. In particular, it was developed to compare the predictions of low-energy Supersymmetry to data both from existing "low-energy" data (LE) and from future colliders, the LHC and eventually a linear electron-positron collider (LC). Here we present a new study using data from existing measurements, future LHC measurements at different integrated luminosities and measurements from a future LC [3]. Similar and related studies can be found in [4, 5, 6, 7]. The models tested are minimal Supergravity (mSugra) with five parameters $m_{1 / 2}, m_{0}, A_{0}, \tan \beta$, and $\operatorname{sgn}(\mu)$ and Gauge Mediated SUSY Breaking (GMSB) with six parameters $\Lambda, M_{\text {mess }}, C_{\text {grav }}, \tan \beta, \operatorname{sgn}(\mu)$, and $N_{5}$. We also investigate a phenomenological Minimal Supersymmetric Standard Model with 18 free parameters (MSSM-18) where no explicit high-scale structure is assumed. R-Parity and minimal flavour violation is always assumed conserved.

Fittino employs a global $\chi^{2}$ function which fully accounts for correlations between the input measurements. The $\chi^{2}$ hyper-surface is scanned and likelihood-maps of the parameter space are obtained using Markov-Chain-Monte-Carlo techniques. Also, global $\chi^{2}$ minimization is performed using a simulated-annealing algorithm combined with Toy-Monte-Carlo techniques for uncertainty determination of the parameters and their correlations.

\section{Constraints from existing measurements}

The precise measurements of low-energy observables, in particular the anomalous magnetic moment of the muon $(g-2)_{\mu}$ and various observables derived from $B$ - and $K$-Meson decays allow us to constrain the allowed SUSY parameter space when being confronted with precise theoretical calculations at the loop level [6]. We also investigate how the cosmologically observed dark matter density constrains the SUSY parameter space by comparing it to the expected relic density of the lightest SUSY particle. Within the mSugra model, a clear preference for parameters is observed which predict a rather light SUSY particle spectrum. Most SUSY particles are predicted to lie below $1 \mathrm{TeV}$ in mass. This spectrum, together with the allowed one- and two-sigma regions is shown in Fig. 1 (left). The parameters for the best fit of the mSugra model with $\operatorname{sgn}(\mu)$ fixed to +1 are $\tan \beta=13.2 \pm 7.2, M_{1 / 2}=331.5 \pm 86.6 \mathrm{GeV}, M_{0}=76.2+79.2-29.1 \mathrm{GeV}$, and $A_{0}=$ $383.8 \pm 647 \mathrm{GeV}$ with $\chi^{2} /$ n.d.f. $=20.6 / 22$. $\operatorname{sgn}(\mu)=+1$ is preferred over $\operatorname{sgn}(\mu)=-1$, mainly due to the measured value of $(g-2)_{\mu}$ being larger than its SM prediction, where the contribution from hadronic vacuum polarization is evaluated from $e^{+} e^{-}$collision data. Similarly good fits can be obtained within GMSB while for MSSM-18 there are too many free parameters to provide a stable fit. The omission of the dark matter density as input yields somewhat larger allowed regions for the sparticle masses within mSugra but central values are not shifted significantly. If $\tau$-decay data are employed to extract the hadronic vacuum polarization, central values of the fit do not shift strongly but the error bands increase significantly. If, instead, $(g-2)_{\mu}$ is set to its SM value the allowed mass ranges increase further and central value move upwards. Colour-neutral sparticles are still constrained to lie below $\approx 2 \mathrm{TeV}$ and coloured sparticles below $3.5 \mathrm{TeV}$ at the $95 \%$ confidence 
level. If both dark matter and $(g-2)_{\mu}$ are removed from the inputs, no meaningful constraints can be derived any longer.
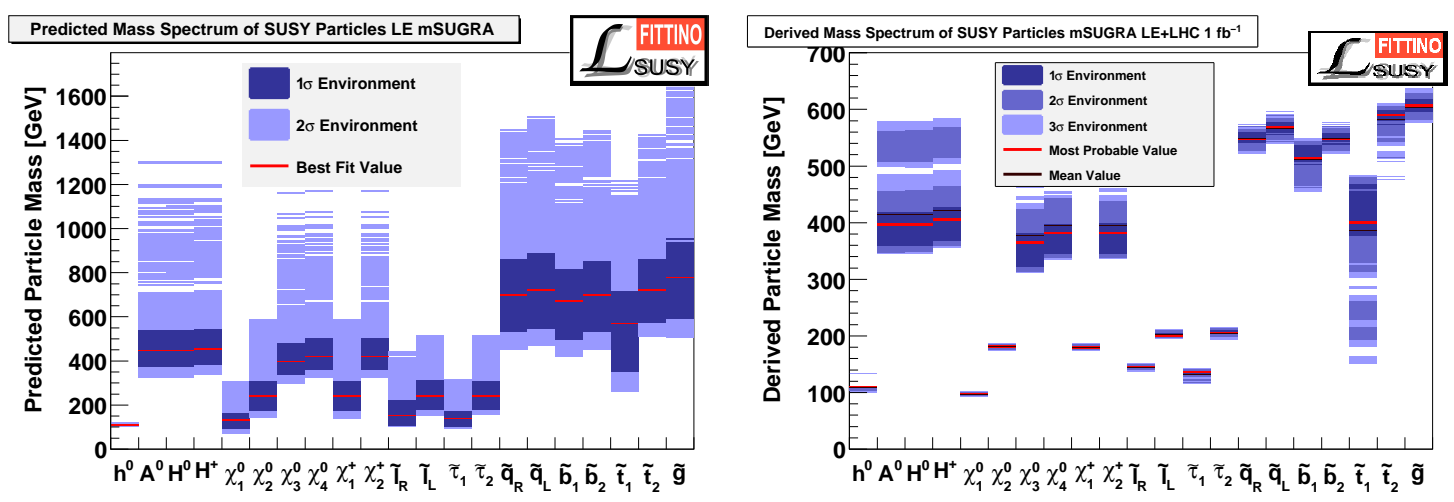

Figure 1: Left: SUSY mass spectrum consistent with the existing low-energy measurements for the mSugra model. Right: SUSY mass spectrum consistent with the existing low-energy measurements together with the expected LHC measurements at $\mathscr{L}^{\text {int }}=1 \mathrm{fb}^{-1}$ for the mSUGRA model.

\section{Prospects for LHC and ILC}

For the collider predictions SPHENO [8] is used. The parameters of the best fit point from LE data are similar to a well-studied SUSY benchmark point, SPS1a [9] with values $\tan \beta=10$, $M_{1 / 2}=250 \mathrm{GeV}, M_{0}=100 \mathrm{GeV}$, and $A_{0}=-100 \mathrm{GeV}$. It is therefore justified to employ the sensitivity studies of the LHC experiments ATLAS and CMS as well as studies carried out within [10] and to investigate the parameter precision as a function of the integrated luminosity at the LHC. Measurements at the LHC, depending on the integrated luminosity, include various characteristic kinematic edges from squark-neutralino decay chains as well as the mass of the lightest Higgs boson. For a full list see [3]. The expected precisions on mSugra parameters for three different integrated luminosities are given in Table 1. The inclusion of LE data into the fit helps in improving the precision in particular on $\tan \beta$ and $A_{0}$ for an LHC integrated luminosity of $1 \mathrm{fb}^{-1}$ while the accuracy is dominated by the future LHC measurements for higher luminosities.

\begin{tabular}{lrrrr}
\hline & \multicolumn{4}{c}{ Uncertainties } \\
Parameter & SPS1a & $1 \mathrm{fb}^{-1}$ & $10 \mathrm{fb}^{-1}$ & $300 \mathrm{fb}^{-1}$ \\
\hline $\operatorname{sign}(\mu)$ & +1 & & & \\
$\tan \beta$ & 10 & 3.7 & 0.84 & 0.35 \\
$M_{1 / 2}(\mathrm{GeV})$ & 250 & 6.7 & 1.2 & 0.30 \\
$M_{0}(\mathrm{GeV})$ & 100 & 4.2 & 2.1 & 0.39 \\
$A_{0}(\mathrm{GeV})$ & -100 & 742.1 & 52.9 & 11.1 \\
\hline
\end{tabular}

Table 1: Expected uncertainties on mSUGRA parameters for integrated LHC luminosities of $1 \mathrm{fb}^{-1}, 10 \mathrm{fb}^{-1}$ and $300 \mathrm{fb}^{-1}$.

In order to fit future LHC measurements, fixed assignments of certain observed features (e.g. kinematic edges) have to be made to the underlying SUSY process which produces them. So 
far, such assignments had to be made ad-hoc. We propose a new method [3] to include such assignment ambiguities into the fit. For each model point, all possible ambiguities are tested and the one leading to the smallest $\chi^{2}$-contribution is included into the global $\chi^{2}$. With this method, parameter uncertainties can be determined without the need to make such ad-hoc assumptions.

Since the mechanism of SUSY breaking is unknown, it is the task of future colliders, the LHC and the LC, to derive information about high-scale mass patterns from the measurements themselves. In order to achieve this in a "bottom-up" approach, the EW-scale MSSM parameters have to be measured with the least possible number of assumptions. We studied how well the parameters of an 18-parameter MSSM Lagrangian (MSSM-18) can be measured, which only assumes universality of the first and second generation mass parameters and the absence of flavour-non-diagonal or CP-violating terms. Since many experimental constraints are needed to determine 18 parameters, this task may be only possible for large integrated luminosities at the LHC and by also including LE measurements. A stable fit can be obtained for $300 \mathrm{fb}^{-1}$ of LHC data where most parameters can be obtained to the $10 \%$-level with the exception of the mixing parameters of the third generation and the mass parameter for the CP-odd Higgs boson, $m_{A}$. In order to achieve a precision suitable for the precise extrapolation of the mass parameters to a high scale, measurements from a Linear Collider are indispensable. Also, to predict the neutralino relic density within the MSSM-18 to precision comparable to that of future determinations of the dark matter density in the Universe, the Linear Collider is needed.

\section{Conclusions}

We presented a study of the present and future constraints on the parameter space of SUSY models. Currently existing measurements with largest impact are the anomalous magnetic moment of the muon and the dark matter density in the Universe which point towards rather light SUSY particles within the mSugra and GMSB scenarios. The preferred scenarios are accessible at the LHC at an early stage. For an initial bottom-up determination of MSSM parameters, ultimate LHC luminosity is required. Precision determination at the percent level is only possible at the future Linear Collider.

\section{References}

[1] P. Bechtle, K. Desch and P. Wienemann, Comput. Phys. Commun. 174 (2006) 47 [arXiv:hep-ph/0412012].

[2] P. Bechtle, K. Desch, W. Porod and P. Wienemann, Eur. Phys. J. C 46 (2006) 533 [arXiv:hep-ph/0511006].

[3] P. Bechtle, K. Desch, M. Uhlenbrock and P. Wienemann, arXiv:0907.2589 [hep-ph].

[4] R. Lafaye, T. Plehn, M. Rauch and D. Zerwas, Eur. Phys. J. C 54 (2008) 617 [arXiv:0709.3985 [hep-ph]].

[5] S. S. AbdusSalam, B. C. Allanach, F. Quevedo, F. Feroz and M. Hobson, arXiv:0904.2548 [hep-ph].

[6] O. Buchmueller et al., JHEP 0809 (2008) 117 [arXiv:0808.4128 [hep-ph]].

[7] O. Buchmueller et al., arXiv:0907.5568 [hep-ph]. 
[8] W. Porod, Comput. Phys. Commun. 153 (2003) 275 [arXiv:hep-ph/0301101].

[9] B. C. Allanach et al., in Proc. of the APS/DPF/DPB Summer Study on the Future of Particle Physics (Snowmass 2001) ed. N. Graf, Eur. Phys. J. C 25 (2002) 113 [arXiv:hep-ph/0202233].

[10] G. Weiglein et al. [LHC/LC Study Group], Phys. Rept. 426 (2006) 47 [arXiv:hep-ph/0410364]. 